\title{
FINITE JET DETERMINATION OF LOCAL CR AUTOMORPHISMS THROUGH RESOLUTION OF DEGENERACIES*
}

\author{
BERNHARD LAMEL ${ }^{\dagger}$ AND NORDINE MIR $\ddagger$
}

Dedicated to M. Salah Baouendi on the occasion of his seventieth birthday

\begin{abstract}
Let $M \subset \mathbb{C}^{N}$ be a connected real-analytic hypersurface whose Levi form is nondegenerate at some point. We prove that for every point $p \in M$, there exists an integer $k=k(M, p)$ such that germs at $p$ of local real-analytic CR automorphisms of $M$ are uniquely determined by their $k$-jets (at $p$ ). To prove this result we develop a new technique that can be seen as a resolution of the degeneracies of $M$. This procedure consists of blowing up $M$ near an arbitrary point $p \in M$ regardless of its minimality or nonminimality; then, thanks to the blow-up, the original problem can be reduced to an analogous one for a very special class of nonminimal hypersurfaces for which one may use known techniques to prove the finite jet determination property of its CR automorphisms.
\end{abstract}

Key words. Finite jet determination, CR automorphism, blow-up, nonminimal hypersurface

AMS subject classifications. 32H02, 32H12, 32V05, 32V15, 32V20, 32V35, 32V40

1. Introduction. This paper is concerned with the finite jet determination problem for germs of CR automorphisms of real-analytic hypersurfaces in complex space. Our main motivation is the following conjecture that essentially goes back to the recent work of Baouendi, Ebenfelt and Rothschild [1]:

CONJECTURE 1.1. Let $M \subset \mathbb{C}^{n+1}$ be a connected real-analytic holomorphically nondegenerate hypersurface, $n \geq 1$. Then for every $p \in M$, there exists a positive integer $k=k(M, p)$ such that germs at $p$ of local real-analytic $C R$ automorphisms of $M$ are uniquely determined by their $k$-jets at $p$.

Let us recall that a (connected) holomorphically nondegenerate real hypersurface is a real hypersurface for which there is no germ of a nontrivial holomorphic vector field tangent to an open piece of $M$ (this notion was introduced by Stanton [32]). A solution to the above conjecture would provide a completely satisfactory local CR version for real-analytic hypersurfaces of $\mathbb{C}^{n+1}$ of the classical uniqueness theorem of H. Cartan [11] stating that holomorphic self-automorphisms of bounded domains in $\mathbb{C}^{n+1}$ are uniquely determined by their 1 -jet at any point of the source domain. Indeed, holomorphic nondegeneracy appears to be the "natural" obstruction to finite jet determination, the necessity of the condition being observed in [1].

Much progress has been made in recent years toward the solution to the above mentioned conjecture and a number of important cases have been settled. Historically, the first case considered was when the given hypersurface has everywhere nondegenerate Levi-form. This was solved by E. Cartan [9, 10], Tanaka [33] and Chern-Moser [12], as a consequence of their solution to the biholomorphic equivalence problem. Furthermore, in that setting unique determination by 2 -jets holds at every point.

\footnotetext{
*Received September 1, 2006; accepted for publication March 15, 2007.

${ }^{\dagger}$ Universität Wien, Fakultät für Mathematik, Nordbergstrasse 15, A-1090 Wien, Österreich (lamelb@member.ams.org). The first author was supported by the FWF, Projekt P17111.

¥Université de Rouen, Laboratoire de Mathématiques Raphaël Salem, UMR 6085 CNRS, Avenue de l'Université, B.P. 12, 76801 Saint Etienne du Rouvray, France (Nordine.Mir@univ-rouen.fr). The research of the second author was supported in part by the French National Agency for Research (ANR), Project Resonances (programme blanc).
} 
In order to follow the lines of the most recent developments related to Conjecture 1.1, it is relevant to understand the structure of a real-analytic holomorphically nondegenerate hypersurface and, in particular, all possible types of Levi-degenerate points that such a manifold can have. And indeed such a hypersurface may contain points that degenerate in various possible ways. One first possible situation is given by the case of a holomorphically nondegenerate real-analytic hypersurface that is everywhere Levi-degenerate. (This may only happen for $n \geq 2$.) The typical example that illustrates this situation is the tube in $\mathbb{C}^{3}$ over the light cone that is given by the set of smooth points of the real-algebraic variety $V=\left\{\left(z_{1}, z_{2}, z_{3}\right) \in \mathbb{C}^{3}:\left(\operatorname{Re} z_{1}\right)^{2}=\right.$ $\left.\left(\operatorname{Re} z_{2}\right)^{2}+\left(\operatorname{Re} z_{3}\right)^{2}\right\}$. For this type of hypersurfaces, neither the results nor the techniques used in the Levi-nondegenerate case are available. This led Baouendi, Huang, Ebenfelt and Rothschild to introduce a finer notion of nondegeneracy, called finite nondegeneracy $[5,2]$. Finite nondegeneracy is more general than Levi-nondegeneracy (e.g. the tube of the light cone is everywhere finitely nondegenerate); the finite jet determination problem for the class of finitely nondegenerate hypersurfaces was solved, among other things, in [1]. This however does not solve Conjecture 1.1 since a connected holomorphically nondegenerate real-analytic hypersurface in general only satisfies the finite nondegeneracy condition at every point of a Zariski open subset [2]. Therefore, the results of [1] provide a solution to Conjecture 1.1 for all points $p \in M$ outside a certain proper real-analytic subvariety $\Sigma$ of $M$.

The above mentioned set $\Sigma \subset M$ consists in a certain sense of the most degenerate points that a hypersurface $M$ as in Conjecture 1.1 may have. In order to deal with this thin set of points, one usually stratifies the set $\Sigma$ into a real-analytic subset $\Sigma_{1}$ and $\Sigma_{2}=\Sigma \backslash \Sigma_{1}$; the set $\Sigma_{1}$ consists of the points in $\Sigma$ through which there passes a complex hypersurface of $\mathbb{C}^{N}$ entirely contained in $M$ (i.e. the set of nonminimal points), the set $\Sigma_{2}$ being therefore the set of minimal points of $\Sigma$. The set $\Sigma_{1}$ consists more precisely of a locally finite disjoint union of complex hypersurfaces of $\mathbb{C}^{N}$ contained in $M$. The stratification comes from the fact that points in $\Sigma_{1}$ and $\Sigma_{2}$ degenerate in different manners. Furthermore, and most importantly, there is a very convenient machinery introduced by Baouendi, Ebenfelt and Rothschild, the socalled Segre set technique (see e.g. [31]), that has been extremely useful in the study of mapping problems but is available only at minimal points. Using, among other tools, this technique, Baouendi, Rothschild and the second author [6] were able to prove Conjecture 1.1 for all minimal points $p \in M$. This constitutes the most general result to date toward the solution of the conjecture for arbitrary $n$. Hence to complete the proof of Conjecture 1.1, it remains to deal with the points lying in the remaining subvariety $\Sigma_{1}$.

In the two-dimensional case, Ebenfelt, Zaitsev and the first author [16] were recently able to treat this set $\Sigma_{1}$ successfully, which provided a complete solution to Conjecture 1.1 for $n=1$. (Note that in that case, the holomorphic nondegeneracy assumption on the hypersurface $M$ is in fact equivalent to its Levi-nonflatness.) In order to prove the finite jet determination property at every point lying on the subvariety $\Sigma_{1}$, the authors of [16] developed a new approach, firstly initiated by Ebenfelt [14] in the study of the regularity of CR mappings, which, roughly speaking, consists of reducing the original problem to studying the unique jet determination of solutions of certain singular systems of differential equations. This approach strongly contrasts with that used to deal with the set $\Sigma_{2}$ of minimal points in e.g. [3,6] and, therefore, up to now, two different approaches have been used to study the finite jet determination problem according to the minimality or nonminimality of the base point. 
In this paper, focusing on the class of generically Levi-nondegenerate real-analytic hypersurfaces, we provide a unified approach to study the finite jet determination problem and, as a consequence, we give a solution to Conjecture 1.1 for this class.

Our main result is the following.

THEOREM 1.2. Let $M \subset \mathbb{C}^{n+1}$ be a connected real-analytic hypersurface whose Levi form is nondegenerate at some point. Then for every point $p \in M$, there exists an integer $k=k(M, p)$ such that germs at $p$ of local real-analytic CR automorphisms of $M$ are uniquely determined by their $k$-jets at $p$.

In Theorem 1.2, even though we assume that the hypersurface contains one (and therefore a dense open subset of) Levi-nondegenerate point(s), there are many Levidegeneracies that the hypersurface may have. Indeed, the class of generically Levinondegenerate real-analytic hypersurfaces provides a natural generalization to $(n+1)$ dimensional complex euclidean space of the class of real-analytic Levi-nonflat hypersurfaces in $\mathbb{C}^{2}$. Consequently, Theorem 1.2 may be seen as a generalization of the mentioned result of [16]. On the other hand, this class also contains the important class of real-analytic hypersurfaces containing no analytic discs for which the finite jet determination property is already known to hold in view of the results of [3] and for which the methods of this paper offer a completely different new proof.

As explained above, the only remaining set $\Sigma_{1} \subset M$ to be dealt with in Theorem 1.2 is that of nonminimal points. However, our proof applies at every point of a hypersurface $M$ satisfying the required conditions regardless of the minimality or nonminimality of the base point. In that respect, our approach is new and differs from the previous ones.

Our proof consists of blowing up a hypersurface $M$ satisfying the assumptions of Theorem 1.2 near an arbitrary point $p \in M$. In the preimage of the blow-up of $M$, we will obtain a nonminimal real-analytic hypersurface $\widehat{M} \subset \mathbb{C}^{N}$ through the origin (Proposition 5.1) which has the property that all local real-analytic CR automorphisms of $M$ can be lifted to CR automorphisms of $\widehat{M}$ provided their $k$-jet at $p$ coincides with that of the identity mapping for $k$ sufficiently large (Proposition 5.2). The finite jet determination property of $M$ near $p$ will then be reduced to the same property for the constructed nonminimal hypersurface $\widehat{M}$. Furthermore, from our construction, we will get an explicit normal form of $\widehat{M}$ near the origin, which itself follows from an explicit normal form for $M$ near $p$ (given in Proposition 4.3) and the explicit form of the blow-up. The obtained explicit normal form of the nonminimal hypersurface $\widehat{M}$ will allow us to use the known techniques and results of $[14,16]$ to conclude that $\widehat{M}$ has the desired finite jet determination property.

Though the content of this paper is focused on Conjecture 1.1, we should mention that there has been recently a lot of work on several different aspects of the finite jet determination of $\mathrm{CR}$ maps. In addition to the papers already mentioned above, we also refer the reader to the papers [7, 28, 8, 19, 13, 23, 22, 21, 15, 20, 24, 27, 25] and the surveys $[4,34,31]$ for more detailed discussions on these various aspects.

The paper is organized as follows. After settling the notation used throughout the paper in $\S 2$, we introduce in $\S 3$ a special class of (germs of) nonminimal realanalytic hypersurfaces of $\mathbb{C}^{n+1}$ and prove the finite jet determination of their local CR automorphisms by following mainly the arguments of $[14,16]$. Such a result will be of fundamental importance at the end of the paper since we will show through $\S 4-$ $\S 5$ that the study of the finite jet determination problem for local CR automorphisms between real-analytic hypersurfaces satisfying the conditions of Theorem 1.2 can be 
reduced to that of $\mathrm{CR}$ automorphisms of the special class of nonminimal hypersurfaces defined in $\S 3$. Let us also mention that $\S 3$ and $\S 4-\S 5$ are completely independent.

2. Notation. Throughout the paper, given positive integers $r, k, q$ we denote by $J_{0,0}^{r}\left(\mathbb{C}^{k}, \mathbb{C}^{q}\right)$ the jet space of order $r$ of germs of local holomorphic maps $h:\left(\mathbb{C}^{k}, 0\right) \rightarrow$ $\left(\mathbb{C}^{q}, 0\right)$; the $r$-jet of $h$ at 0 is denoted by $j_{0}^{r} h$.

For every real-analytic hypersurface $M \subset \mathbb{C}^{n+1}$ and for every point $p \in M$, we denote by $\operatorname{Aut}(M, p)$ the group of germs at the origin of real-analytic CR automorphisms of $M$ or, equivalently, of biholomorphisms of $\mathbb{C}^{n+1}$ fixing $p$ and sending (the germ of) $M$ into itself. In this paper, the choice of the reference point $p$ will always be fixed (and we will usually have $p$ equal to the origin in $\mathbb{C}^{n+1}$ ). Recall that a choice of local holomorphic coordinates $(z, w) \in \mathbb{C}^{n} \times \mathbb{C}$, vanishing at $p$, is called normal if $M$ can be locally given in these coordinates by an equation of the form

$$
w=Q(z, \bar{z}, \bar{w})
$$

for some holomorphic function $Q=Q(z, \chi, \tau)$ defined near the origin in $\mathbb{C}^{2 n+1}$ satisfying

$$
Q(z, 0, \tau)=Q(0, \chi, \tau)=\tau, \quad Q(z, \chi, \bar{Q}(\chi, z, w))=w,
$$

where here, and for the remainder of the paper, we denote by $\bar{h}$ the series obtained by taking complex conjugates of the coefficients of the power series $h$. It is wellknown that such a choice of normal coordinates always exists (see e.g. [2]). In the remainder of the paper, we will often use the single notation $(z, w)$ for a choice of normal coordinates that may change in various propositions and lemmas.

3. Finite jet determination for a special class of nonminimal hypersurfaces. The appropriate class of nonminimal hypersurfaces under study in this section is given in the following.

Definition 3.1. A germ of a real-analytic hypersurface $M \subset \mathbb{C}^{n+1}$ through the origin will be called a good nonminimal hypersurface if there exists a choice of normal coordinates $(z, w) \in \mathbb{C}^{n} \times \mathbb{C}$ such that $M$ is given by an equation of the form (2.1) with the function $Q$ satisfying

$$
Q(z, \chi, \tau)=\tau+\tau^{m} i\langle z, \chi\rangle+\tau^{m+1} \Theta(z, \chi, \tau),
$$

where $m$ is some positive integer, $\langle z, \chi\rangle=\epsilon_{1} z_{1} \chi_{1}+\cdots+\epsilon_{n} z_{n} \chi_{n}, \epsilon_{j} \in\{-1,1\}$ and $\Theta$ is some holomorphic function near $0 \in \mathbb{C}^{2 n+1}$.

Let us note that a good nonminimal hypersurface $M$ is of $m$-infinite type (in the sense of $[29,14]$ ) and that it can also be defined in normal coordinates by a real equation of the form

$$
\operatorname{Im} w=\varphi(z, \bar{z}, \operatorname{Re} w)=(\operatorname{Re} w)^{m}\langle z, \bar{z}\rangle+O\left((\operatorname{Re} w)^{m+1}\right),
$$

where $\varphi$ is some real-analytic function near the origin in $\mathbb{R}^{2 n+1}$. For this and further notions and standard facts about nonminimal (or equivalently, infinite type) hypersurfaces, we refer the reader to the papers $[29,14,26]$.

The goal of this section is to provide a proof of the following result:

Proposition 3.2. Let $M \subset \mathbb{C}^{n+1}$ be a germ through the origin of a good nonminimal real-analytic hypersurface. Then $(M, 0)$ has the finite jet determination property: 
There exists a positive integer $K$ such that if $H:\left(\mathbb{C}^{n+1}, 0\right) \rightarrow\left(\mathbb{C}^{n+1}, 0\right)$ is a local biholomorphism sending $M$ into itself with the same $K$-jet at the origin as that of the identity mapping, then $H$ is the identity.

REMARK 3.3. At this point, we should mention that the proof of Proposition 3.2 could be achieved by essentially combining several arguments and results from the papers $[14,16]$. For the reader's convenience, we will give a complete proof of this result here, which avoids referring to too many results in the literature. As in [14], we shall derive a singular complete system of differential equations for the automorphisms of the hypersurfaces considered in Proposition 3.2. Due to the special normal form of the hypersurfaces, the construction of this complete system has the advantage to be shorter and substantially easier than in [14]; in particular, we are not forced to work on the tangent bundle of the manifolds in this paper. A suitable adaptation of the arguments in [16] then gives the desired finite jet determination property for the hypersurfaces under consideration.

For the proof of Proposition 3.2, we fix a choice of normal coordinates $(z, w)$ for $(M, 0)$ satisfying the conditions of Definition 3.1, and in these coordinates, we split every $H \in \operatorname{Aut}(M, 0)$ as follows $H=(F, G)$. We also use the notation used in the definition. We start with the following known fact.

Lemma 3.4. Let $M, m$ and $H=(F, G)$ be as given above. Then

$$
G_{w^{\ell}}(z, 0)=G_{w^{\ell}}(0) \in \mathbb{R}, \quad \ell \leq m
$$

Proof. Since $H$ sends $M$ into itself, we have the identity

$$
G(z, Q(z, \chi, \tau))=Q(F(z, Q(z, \chi, \tau)), \bar{F}(\chi, \tau), \bar{G}(\chi, \tau)),
$$

which holds for $(z, \chi, \tau) \in \mathbb{C}^{2 n+1}$ close enough to $(0,0,0)$. Since $M$ is a good nonminimal hypersurface and since $H$ is invertible, we have the following relations

$$
G(z, Q(z, \chi, \tau))=G(z, \tau)+i \tau^{m} G_{w}(z, \tau)\langle z, \chi\rangle+O\left(\tau^{m+1}\right)
$$

and

$Q(F(z, Q(z, \chi, \tau)), \bar{F}(\chi, \tau), \bar{G}(\chi, \tau))=\bar{G}(\chi, \tau)+i \bar{G}(\chi, \tau)^{m}\langle F(z, 0), \bar{F}(\chi, 0)\rangle+O\left(\tau^{m+1}\right)$.

Therefore expanding both sides of (3.3) as power series in $\tau$, and comparing the coefficients of $\tau^{\ell}$ for $\ell<m$, we get

$$
G_{w^{\ell}}(z, 0)=\bar{G}_{w^{\ell}}(\chi, 0)
$$

thus, (3.2) follows for $\ell<m$. Comparing the coefficient of $\tau^{m}$ in (3.3), we also obtain

$$
G_{w^{m}}(z, 0)+G_{w}(z, 0) i\langle z, \chi\rangle=\bar{G}_{\tau^{m}}(\chi, 0)+i \bar{G}_{\tau}(\chi, 0)^{m}\langle F(z, 0), \bar{F}(\chi, 0)\rangle ;
$$

Now setting $\chi=0$ we obtain (3.2) for $\ell=m$. This completes the proof of the lemma.

Keeping the notation defined above, for every $H \in \operatorname{Aut}(M, 0)$, thanks to Lemma 3.4 we may write $G(z, w)=P(w)+w^{m} G_{2}(z, w)$ where $P(w)=$ $\sum_{j=1}^{m-1} \frac{G_{w^{j}}(0)}{j !} w^{j}$. Note that $P(w)=\bar{P}(w)$ and that if $m>1, P(w)=w \tilde{P}(w)$ with 
$\tilde{P}(0) \neq 0$ (for $m=1$, we have $\tilde{P} \equiv 0$ ). When $H$ is the identity mapping, the above splitting is written as follows $\operatorname{Id}=\left(F^{0}, G^{0}\right)$ with $G^{0}(z, w)=P^{0}(w)+w^{m} G_{2}^{0}(z, w)$. (Note that $G_{2}^{0} \equiv 0$ if $m>1$ and $G_{2}^{0} \equiv 1$ if $m=1$.) Observe furthermore that for every $H \in \operatorname{Aut}(M, 0)$, we may write $P(\bar{w})-P(w)=(\bar{w}-w) \mathcal{Q}\left(w, \bar{w}, j_{0}^{m-1} G\right)$ and $\tilde{P}(w)=\mathcal{T}\left(w, j_{0}^{m-1} G\right)$ where $\mathcal{Q}, \mathcal{T}$ are universal polynomials in all their arguments.

We will first proceed to derive a singular complete system for the mapping $V:=$ $\left.\left(F, G_{2}\right)\right|_{M}$ associated to any $H \in \operatorname{Aut}(M, 0)$ provided the $(m+2)$-jet of $H$ (at 0$)$ is sufficiently close to that of the identity mapping. In the complete system, we will keep the coefficients of $P$, or equivalently the $(m-1)$-jet of $G$ at 0 , as parameters. To this end, we need to introduce some further notation. Since $M$ may also be defined near the origin by an equation of the form (3.1), we may use $t:=(z, \bar{z}, s)$ where $s=\operatorname{Re} w$ as real-analytic coordinates for $M$ near 0 and we also write $\theta:=(\operatorname{Re} z, \operatorname{Im} z)$. The precise singular complete system we need is given in the following lemma.

LEMma 3.5. In the above setting, there exists a real-analytic map $\Upsilon$ defined in a neighbourhood of the point $\varpi_{0}:=\left(0, j_{0}^{m-1} G^{0},\left(\left(\left(s^{m} \partial_{s}^{c}\right) \partial_{\theta}^{d} V^{0}\right)(0)\right)_{c+|d| \leq 2}\right)$ such that for every $H \in \operatorname{Aut}(M, 0)$ with $j_{0}^{m+2} H$ sufficiently close to $j_{0}^{m+2} \mathrm{Id}$, the following identity holds for $(\theta, s)$ sufficiently close to $0 \in \mathbb{R}^{2 n+1}$ :

$$
\left(\left(s^{m} \frac{\partial}{\partial s}\right)^{a} \frac{\partial^{|b|}}{\partial \theta^{b}} V\right)_{a+|b| \leq 3}=\Upsilon\left(\theta, s, j_{0}^{m-1} G,\left(\left(s^{m} \frac{\partial}{\partial s}\right)^{c} \frac{\partial^{|d|}}{\partial \theta^{d}} V\right)_{c+|d| \leq 2}\right) .
$$

Here $V=\left.\left(F, G_{2}\right)\right|_{M}$ and $V^{0}:=\left.\left(F^{0}, G_{2}^{0}\right)\right|_{M}$ are as defined above and $t=(\theta, s)$ are used as local coordinates for $M$ near 0 .

Proof. For every $H \in \operatorname{Aut}(M, 0)$, since $H$ sends $M$ into itself, we have the following "basic equality"

$$
\begin{aligned}
G(z, w)= & Q(F(z, w), \bar{F}(\bar{z}, \bar{w}), \bar{G}(\bar{z}, \bar{w})) \\
= & \bar{G}(\bar{z}, \bar{w})+i(\bar{G}(\bar{z}, \bar{w}))^{m}\langle F(z, w), \bar{F}(\bar{z}, \bar{w})\rangle \\
& +(\bar{G}(\bar{z}, \bar{w}))^{m+1} \Theta(F(z, w), \bar{H}(\bar{z}, \bar{w})),
\end{aligned}
$$

which is valid for $(z, w) \in M$ close to 0 . Using the local real-analytic coordinates $t$, then (3.5) is valid for all $t$ in a neighbourhood of 0 in $\mathbb{R}^{2 n+1}$, where we now think of $z, w, \bar{z}, \bar{w}$ as real-analytic functions of $t$. Since $M$ is of $m$-infinite type, $m \geq 1$, the functions $w=w(t)$ and $\bar{w}=\overline{w(t)}$ have the following properties:

(P1) The function $A(t):=\overline{w(t)} / w(t)$ is real-analytic (near 0 ) and its value at the origin given by 1 ;

(P2) We have $w-\bar{w}=O\left(s^{m}\right)$; this implies that the function $B(t):=(\overline{w(t)}-$ $w(t)) /(w(t))^{m}$ is also real-analytic near 0 .

We rewrite the basic equation (3.5) as

$$
\begin{aligned}
w^{m} G_{2}(z, w)= & (P(\bar{w})-P(w))+\bar{w}^{m} \bar{G}_{2}(\bar{z}, \bar{w}) \\
& +i\left(\bar{w}^{m}\right)\left(\overline{\tilde{P}}(\bar{w})+\bar{w}^{m-1} \bar{G}_{2}(\bar{z}, \bar{w})\right)^{m}\langle F(z, w), \bar{F}(\bar{z}, \bar{w})\rangle \\
& +\bar{w}^{m+1}\left(\overline{\tilde{P}}(\bar{w})+\bar{w}^{m-1} \bar{G}_{2}(\bar{z}, \bar{w})\right)^{m+1} \Theta(F(z, w), \bar{F}(\bar{z}, \bar{w}), \bar{w} \tilde{\tilde{P}}(\bar{w}) \\
& \left.+\bar{w}^{m} \bar{G}_{2}(\bar{z}, \bar{w})\right),
\end{aligned}
$$

which holds for all $t \in \mathbb{R}^{2 n+1}$ sufficiently close to 0 . In what follows, if the variables are not written, it is understood that barred functions have $(\bar{z}, \bar{w})$ as their arguments, 
while unbarred functions have $(z, w)$ as arguments. It is also understood that we still view the functions $(z, \bar{z}, w, \bar{w})$ as functions of $t$, but we do not write it in order to avoid too heavy equations.

In view of Properties (P1) and (P2), we may divide (3.6) by $w^{m}$ and obtain an identity of the form

$$
\begin{aligned}
G_{2}= & B(t) \mathcal{Q}\left(w, \bar{w}, j_{0}^{m-1} G\right)+(A(t))^{m} \bar{G}_{2} \\
& +i(A(t))^{m}\left(\overline{\mathcal{T}}\left(\bar{w}, j_{0}^{m-1} G\right)+\bar{w}^{m-1} \bar{G}_{2}\right)^{m}\langle F, \bar{F}\rangle \\
& +\bar{w}(A(t))^{m}\left(\overline{\mathcal{T}}\left(\bar{w}, j_{0}^{m-1} G\right)+\bar{w}^{m-1} \bar{G}_{2}\right)^{m+1} \Theta\left(F, \bar{F}, \bar{w} \overline{\mathcal{T}}\left(\bar{w}, j_{0}^{m-1} G\right)+\bar{w}^{m} \bar{G}_{2}\right),
\end{aligned}
$$

valid for all $t \in \mathbb{R}^{2 n+1}$ sufficiently close to 0 . Notice that we may rewrite the expression

$$
\left(\overline{\mathcal{T}}\left(\bar{w}, j_{0}^{m-1} G\right)+\bar{w}^{m-1} \bar{G}_{2}\right)^{m+1} \Theta\left(F, \bar{F}, \bar{w} \overline{\mathcal{T}}\left(\bar{w}, j_{0}^{m-1} G\right)+\bar{w}^{m} \bar{G}_{2}\right)
$$

in the following form

$$
R\left(t, F, \bar{F}, \bar{G}_{2}, j_{0}^{m-1} G\right)
$$

for some universal function $R$ in all its arguments (depending only on $M$ ) holomorphic in some neighbhourhood of $\{0\} \times \mathbb{C} \times J_{0,0}^{m-1}\left(\mathbb{C}^{n+1}, \mathbb{C}\right) \subset \mathbb{C}^{4 n+1} \times \mathbb{C} \times J_{0,0}^{m-1}\left(\mathbb{C}^{n+1}, \mathbb{C}\right)$.

We now proceed by differentiating the identity

$$
\begin{aligned}
& G_{2}=B(t) \mathcal{Q}\left(w, \bar{w}, j_{0}^{m-1} G\right)+(A(t))^{m} \bar{G}_{2}+ \\
& i(A(t))^{m}\left(\overline{\mathcal{T}}\left(\bar{w}, j_{0}^{m-1} G\right)+\bar{w}^{m-1} \bar{G}_{2}\right)^{m}\langle F, \bar{F}\rangle+\bar{w}(A(t))^{m} R\left(t, F, \bar{F}, \bar{G}_{2}, j_{0}^{m-1} G\right)
\end{aligned}
$$

with respect to the $\mathrm{CR}$ vector fields on $M$. To this end, with our choice of coordinates for $M$, we choose as a basis of the CR structure on $M$ the following vector fields

$$
L_{j}:=\frac{\partial}{\partial \bar{z}_{j}}-\frac{\varphi_{\bar{z}_{j}}}{\varphi_{s}-i} \frac{\partial}{\partial s}, \quad j=1, \ldots, n
$$

In what follows, a multiindex $J$ is a finite sequence $\left(J_{1}, \ldots, J_{\ell}\right) \subset\{1, \ldots, n\}^{\ell}$ and we denote by $|J|:=\ell$ the length of $J$. Then $L^{J}$ (resp. $\bar{L}^{J}$ ) denotes the differential operator $L_{J_{1}} \ldots L_{J_{\ell}}\left(\right.$ resp. $\left.\bar{L}_{J_{1}} \ldots \bar{L}_{J_{\ell}}\right)$.

We now apply $L_{j}$ to $(3.8)$ for every $j=1, \ldots, n$. Since $L_{j} G_{2}=0$, we obtain an equation of the form

$$
0=\Psi_{j}\left(t, F, \bar{F}, \bar{G}_{2}, L_{j} \bar{F}, L_{j} \bar{G}_{2}, j_{0}^{m-1} G\right),
$$

for $t \in \mathbb{R}^{2 n+1}$ close to 0 , where $\Psi_{j}$ is holomorphic in a neighbourhood of $\{0\} \times \mathbb{C} \times \mathbb{C}^{n} \times$ $\mathbb{C} \times J_{0,0}^{m-1}\left(\mathbb{C}^{n+1}, \mathbb{C}\right) \subset \mathbb{C}^{6 n+3} \times J_{0,0}^{m-1}\left(\mathbb{C}^{n+1}, \mathbb{C}\right)$ (and independent of the mapping $H$ ). We denote by $A=\left(A_{1}, \ldots, A_{n}\right)$ the second set of variables appearing in the function $\Psi_{j}$ (and in which the map $F$ appears). We now evaluate the partial derivative of $\Psi_{j}$ with respect to $A_{k}$ at the reference point corresponding to the identity mapping. Using the fact that $L_{j} \bar{w}(0)=0$ and $A(0)=1$, we obtain

$$
\frac{\partial \Psi_{j}}{\partial A_{k}}\left(0,0,0, \bar{G}_{2}^{0}(0), L_{j} \bar{F}^{0}(0), L_{j} \bar{G}_{2}^{0}(0), j_{0}^{m-1} G^{0}\right)=i \epsilon_{k} \delta_{j, k},
$$

where $\delta_{j, k}$ denotes the Kronecker symbol. Thus, we may apply the Implicit Function Theorem to the equations (3.9) for $j=1, \ldots, n$ and conclude that there 
exists a $\mathbb{C}^{n}$-valued holomorphic map $\Phi$ defined in a neighbourhood of $\eta_{0}:=$ $\left(0,\left(\left(L^{\alpha} \bar{F}^{0}(0), L^{\alpha} \bar{G}_{2}^{0}(0)\right)\right)_{|\alpha| \leq 1}, j_{0}^{m-1} G^{0}\right)$ in $\mathbb{C}^{2 n+1} \times \mathbb{C}^{(n+1)^{2}} \times J_{0,0}^{m-1}\left(\mathbb{C}^{n+1}, \mathbb{C}\right)$ such that for every $H \in \operatorname{Aut}(M, 0)$ whose $(m+1)$-jet at 0 is sufficiently close to that of the identity mapping, then

$$
F=\Phi\left(t,\left(L^{\alpha} \bar{F}, L^{\alpha} \bar{G}_{2}\right)_{|\alpha| \leq 1}, j_{0}^{m-1} G\right),
$$

for $t \in \mathbb{R}^{2 n+1}$ close to 0 . Plugging (3.10) into (3.8) we get an analogous equation for $G_{2}$. For every map $H \in \operatorname{Aut}(M, 0)$ as above, recalling that $V=\left.\left(F, G_{2}\right)\right|_{M}$, we get that $V$ satisfies an identity in the neighbourhood of the origin in $\mathbb{R}^{2 n+1}$ of the form

$$
V=\Xi\left(t,\left(L^{\alpha} \bar{V}\right)_{|\alpha| \leq 1}, j_{0}^{m-1} G\right),
$$

where $\Xi$ is a $\mathbb{C}^{n+1}$-valued holomorphic map defined in a neighbourhood of $\eta_{0}$. In what follows, the elements $H \in \operatorname{Aut}(M, 0)$ for which $j_{0}^{m+1} H$ is close enough to $j_{0}^{m+1}$ Id so that (3.11) holds will be called admissible maps.

Having established (3.11), we can now use arguments similar to [14] but somewhat easier and closer to the paper of Han [18] in order to derive the desired singular complete system. For this, we set

$$
S:=s^{m} \frac{\partial}{\partial s},
$$

and note that we have the commutation identities

$$
\left[L_{j}, L_{k}\right]=0, \quad\left[L_{j}, \bar{L}_{k}\right]=a_{j, k} S, \quad\left[L_{j}, S\right]=b_{j} S, \quad j, k=1, \ldots, n,
$$

where the $a_{j, k}$ and the $b_{j}$ are germs of real-analytic functions at $0 \in \mathbb{R}^{2 n+1}$, and $a_{j, j}(0) \neq 0$.

Let us now recall the following fact from [14, Proposition 4.3]: for any multi-index $J$, integer $k \geq 1$, there exist real-analytic functions $b_{q}^{e_{1} \ldots e_{m}}$ near 0 in $\mathbb{R}^{2 n+1}$ such that

$$
\sum_{m=1}^{|J|+k} \sum_{q=0}^{k} b_{q}^{e_{1} \ldots e_{m}} \underbrace{\left.\left[\ldots\left[\bar{L}_{e_{1}} \ldots \bar{L}_{e_{m}}, L_{1}\right], L_{1}\right] \ldots, L_{1}\right]}_{\text {length } q}=\bar{L}^{J} S^{k} .
$$

Here the length of the commutator $\left.\left[\ldots\left[X, Y_{1}\right], Y_{2}\right] \ldots, Y_{q}\right]$ is $q$.

For every integer $j$ and multiindex $J$, we use the notation $\Lambda_{j, J}$ to denote new variables lying in $\mathbb{C}^{n+1}$ and $\lambda$ for a jet in the space $J_{0,0}^{m-1}\left(\mathbb{C}^{n+1}, \mathbb{C}\right)$. Further, for every nonnegative integers $p$ and $q$, we denote by $C_{p, q}\left(\right.$ resp. $\left.\bar{C}_{p, q}\right)$ the set of $\mathbb{C}^{n+1}$ valued holomorphic maps $\mathcal{A}=\mathcal{A}\left(t,\left(\Lambda_{j, J}\right)_{\substack{j+|J| \leq p \\ j \leq q}}, \lambda\right)$ that are polynomials in the variables $\left(\Lambda_{j, J}\right)_{j>0}$ and $\left(\Lambda_{0, J}\right)_{|J|>1}$ with holomorphic coefficients in a neighbourhood of $\omega_{0}:=\left(0,\left(\left(L^{J} \bar{F}^{0}(0), L^{J} \bar{G}_{2}^{0}(0)\right)\right)_{|J| \leq 1}, j_{0}^{m-1} G^{0}\right)$ in $\mathbb{C}^{2 n+1} \times \mathbb{C}^{(n+1)^{2}} \times J_{0,0}^{m-1}\left(\mathbb{C}^{n+1}, \mathbb{C}\right)$ (resp. in a neighbourhood of $\bar{\omega}_{0}$ ).

Applying $\bar{L}_{j}$ to (3.11) and using the fact that $\bar{L}_{j} \bar{V}=0$, we get that for every $j=1, \ldots, n$, there exists a map $\Xi_{j} \in C_{1,1}$ such for all admissible maps $H$

$$
\bar{L}_{j} V=\Xi_{j}\left(t,\left(L^{J} S \bar{V}\right) \underset{\substack{j+|J| \leq 1 \\ j \leq 1}}{ }, j_{0}^{m-1} G\right) .
$$

Due to the commutation identities (3.12), further applications of any vector field $\bar{L}_{k}$ to (3.14) will not increase the order of differentiation of the arguments of the right 
hand side of (3.14), and therefore for every multiindex $E$, there exists $\Xi_{E} \in C_{1,1}$, such that for every admissible map $H$, the following identity holds in a neighbourhood of the origin in $\mathbb{R}^{2 n+1}$

$$
\bar{L}^{E} V=\Xi_{E}\left(t,\left(L^{J} S^{j} \bar{V}\right)_{\substack{j+|J| \leq 1 \\ j \leq 1}}, j_{0}^{m-1} G\right) .
$$

In order to get a similar identity for the map $L^{J} S^{k} V$ for every multiindex $J$ and integer $k$, we note that the commutator identity (3.13) and the fact that $L_{1} V=0$ imply that we just need to apply at most $|J|+k$ vector fields of the family $\left\{\bar{L}_{1}, \ldots, \bar{L}_{n}\right\}$ to (3.11), followed by an application of at most $k$ instances of $L_{1}$. Therefore to get the desired equations for the expressions $L^{J} S^{k} V$ we only need to apply at most $k$ instances of the vector field $L_{1}$ to (3.15). We thus get for every multiindex $E$ the existence of $\Xi_{E, k} \in C_{1+k, 1}$ such that

$$
L_{1}^{k} \bar{L}^{E} V=\Xi_{E, k}\left(\left(t,\left(L^{J} S^{j} \bar{V}\right)_{\substack{j+|J| \leq 1+k \\ j \leq 1}}, j_{0}^{m-1} G\right),\right.
$$

for $t$ close to $0 \in \mathbb{R}^{2 n+1}$ and hence for every $J$ and $k$, there exists $\tilde{\Xi}_{J, k} \in C_{1+k, 1}$ such that for all admissible maps $H$,

$$
\bar{L}^{J} S^{k} V=\tilde{\Xi}_{J, k}\left(\left(t,\left(L^{K} S^{\ell} \bar{V}\right)_{\substack{\ell+|K| \leq 1+k \\ \ell \leq 1}}, j_{0}^{m-1} G\right) .\right.
$$

Taking the complex conjugate of (3.16) for $k=1$ and plugging the resulting equation into (3.16) for $|J|+k \leq 3$, we get for any such $J$ and $k$ a map $\hat{\Xi}_{J, k} \in \bar{C}_{2,1}$ such that for all admissible maps $H$,

$$
\bar{L}^{J} S^{k} V=\hat{\Xi}_{J, k}\left(t,\left(\bar{L}^{K} S^{\ell} V\right)_{\substack{\ell+|K| \leq 2 \\ \ell \leq 1}}, j_{0}^{m-1} G\right),
$$

in a neighbourhood of the origin in $\mathbb{R}^{2 n+1}$. Since $L_{\nu} V=0$ for all $\nu=1, \ldots, n$, rewriting the system (3.17) in the real coordinates $t=(\theta, s)$ we get that each map $V$ as above satisfies a singular complete system of order 3, i.e. that (3.4) is satisfied for every map $V$ corresponding to a map $H \in \operatorname{Aut}(M, 0)$ for which $j_{0}^{m+2} H$ is sufficiently close to $j_{0}^{m+2} \mathrm{Id}$. This completes the proof of Lemma 3.5.

Proof. [Proof of Proposition 3.2] Keeping the previously defined notation, we shall prove that there exists an integer $K \geq(m+4)$ such that if $H \in \operatorname{Aut}(M, 0)$ satisfies $j_{0}^{K} H=j_{0}^{K}$ Id then the complete system satisfied by the corresponding map $V=\left.\left(F, G_{2}\right)\right|_{M}$ (and given in Lemma 3.5) has only $V^{0}$ as a solution, which shows that $H=$ Id.

We start by considering only those $H \in \operatorname{Aut}(M, 0)$ for which $j_{0}^{m+4} H=j_{0}^{m+4} \mathrm{Id}$ and making the following observation. In view of Lemma 3.5 all such maps have their component $V=\left.\left(F, G_{2}\right)\right|_{M}$ that satisfy the same complete system. For all such $V$ 's, the map $V(\theta, 0)$ satifies an ordinary complete system of order 3 (in the variable $\theta$ ), and, therefore, $V(\theta, 0)$ is real-analytically parametrized by its 2 -jet at 0 (see e.g. [13, Proposition 3.54]). Similarly, after differentiating (3.4) with respect to $s \ell$ times, we see that for every integer $\ell$ the maps $V_{s^{\ell}}(\theta, 0)$ satisfy analogously an ordinary complete system of order 3 and, hence, such maps $V_{s^{\ell}}(\theta, 0)$ are also real-analytically parametrized by the $(\ell+2)$-jets of $V$ at 0 .

Next, writing for every $V$ as above

$$
U(\theta, s):=\left(\left(s^{m} \frac{\partial}{\partial s}\right)^{a} \frac{\partial^{|b|}}{\partial \theta^{b}} V\right)_{a+|b| \leq 2}, \quad \mathcal{U}(\theta, s):=U(\theta, s)-U(\theta, 0),
$$


and $U^{0}, \mathcal{U}^{0}$ for the corresponding maps obtained from $V^{0}$, we note that it follows from the observation and the assumption $j_{0}^{m+4} H=j_{0}^{m+4}$ Id that $U(\theta, 0)=U^{0}(\theta, 0)$. It therefore follows from (3.4) that $\mathcal{U}$ fulfills a real-analytic equation of the form

$$
s^{m} \frac{\partial \mathcal{U}}{\partial s}(\theta, s)=\widetilde{\Upsilon}\left(\theta, s, j_{0}^{m-1} G^{0}, \mathcal{U}(\theta, s)+U^{0}(\theta, 0)\right),
$$

where $\widetilde{\Upsilon}$ is real-analytic in a neighbhourhood of $\varpi_{0}$ (where $\varpi_{0}$ is given in Lemma 3.5). Since $\mathcal{U}(\theta, 0)=0$, we are now in a position to apply the determination theorem $[16$, Theorem 5.1] which provides an integer $k$ such that if $\mathcal{U}$ satisfies $\mathcal{U}_{s^{j}}(\theta, 0)=\mathcal{U}_{s^{j}}^{0}(\theta, 0)$ for $j \leq k$, then $\mathcal{U}(\theta, s)=\mathcal{U}^{0}(\theta, s)$. But, again, in view of the observation, the last condition is fulfilled provided the $(k+4)$-jet of $V$ at 0 agrees with that of $V^{0}$. Now setting $K:=m+4+k$, we get the desired result. The proof of the proposition is complete.

4. A normal form for a generically Levi-nondegenerate real-analytic hypersurface. The goal of this section is to provide, for any connected real-analytic hypersurface $M \subset \mathbb{C}^{n+1}$ which is Levi-nondegenerate at some point, a normal form for the hypersurface near any of its points. This normal form will be provided by Proposition 4.3 below.

In the following lemma, the notation $T_{p}^{c} M$ denotes the usual complex tangent space of $M$ at $p$ (see e.g. [2]). The content of the following lemma is well-known, but for the reader's convenience we provide a proof.

Lemma 4.1. Let $M \subset \mathbb{C}^{n+1}$ be a real-analytic hypersurface and $p \in M$. Let $\Gamma$ be a real-analytic curve passing through $p$ that is transverse to $T_{p}^{c} M$. Then there exists a choice of normal coordinates $(z, w) \in \mathbb{C}^{n} \times \mathbb{C}$ for $M$ near $p$, vanishing at $p$, such that the germ at $p$ of $\Gamma$ is given in these coordinates by the germ of the real line $\{(0, s): s \in(\mathbb{R}, 0)\}$.

Proof. We first choose an arbitrary set of normal coordinates $\left(z^{\prime}, w^{\prime}\right) \in \mathbb{C}^{n} \times \mathbb{C}$, vanishing at $p$, so that $M$ is given near the origin by an equation of the form

$$
\operatorname{Im} w^{\prime}=\psi\left(z^{\prime}, \bar{z}^{\prime}, \operatorname{Re} w^{\prime}\right),
$$

with $\psi$ real-analytic in a neighborhood of $0 \in \mathbb{R}^{2 n+1}$ and satisfying $\psi\left(z^{\prime}, 0, \operatorname{Re} w^{\prime}\right)=$ $\psi\left(0, \bar{z}^{\prime}, \operatorname{Re} w^{\prime}\right) \equiv 0$. Let $(\mathbb{R}, 0) \ni t \mapsto \gamma(t) \in\left(\mathbb{C}^{n+1}, 0\right)$ be a parametrization of the curve $\Gamma$ near the origin. Since $\Gamma$ is transverse to $T_{p}^{c} M$ by assumption, we may assume, after reparametrizing the curve if necessary that $\gamma(t)=(\beta(t), \eta(t)) \in \mathbb{C}^{n} \times \mathbb{C}$ with $\eta(t)=t+i \psi(\beta(t), \overline{\beta(t)}, t)$ for $t \in \mathbb{R}$ close enough to the origin. In what follows, we shall complexify the maps $\beta$ and $\eta$ and keep the same notation for the complexified maps. By the implicit function theorem, (4.1) is equivalent to an equation of the form $w^{\prime}=Q^{\prime}\left(z^{\prime}, \bar{z}^{\prime}, \bar{w}^{\prime}\right)$ for some holomorphic function $Q^{\prime}$ near the origin in $\mathbb{C}^{2 n+1}$ satisfying (2.2) (with $Q$ replaced by $Q^{\prime}$ ). Consider the coordinates $(z, w) \in \mathbb{C}^{n} \times \mathbb{C}$ defined by the following holomorphic change of variables:

$$
\left\{\begin{array}{l}
z^{\prime}=z+\beta(w) \\
w^{\prime}=Q^{\prime}(z+\beta(w), \bar{\beta}(w), \bar{\eta}(w)) .
\end{array}\right.
$$

By using the normality of the $\left(z^{\prime}, w^{\prime}\right)$ coordinates and the fact that $\eta(w)=$ $Q^{\prime}(\beta(w), \bar{\beta}(w), \bar{\eta}(w))$ for $w \in \mathbb{C}$ close to 0 (which comes from the fact that $\Gamma \subset M$ ), the reader can verify that the obtained $(z, w)$ coordinates satisfy all the requirements of the lemma. The proof of Lemma 4.1 is complete. $\square$ 
We now go through the construction of the desired normal form for a real-analytic generically Levi-nondegenerate hypersurface. In what follows, for any matrix $U$ with entries in $\mathbb{C}$, we set $U^{*}:=\overline{{ }^{t} U}$, where ${ }^{t} U$ denotes the transpose matrix of $U$. We will make use of the following result that goes back to Rellich [30] (see also [17]):

Proposition 4.2. Let $A(s)$ be a complex-valued $k \times k$ matrix whose entries depend real-analytically on $s \in(a, b) \subset \mathbb{R}$. Assume that $A(s)$ is hermitian for every $s \in(a, b)$. Then there exists $a k \times k$ unitary matrix $U(s)$, depending real-analytically on $s \in(a, b)$ such that the matrix

$$
U(s) A(s) U^{*}(s)
$$

is a diagonal matrix (with real-analytic entries in $s$ ).

Proposition 4.2 is the main ingredient for the construction of the following normal form.

Proposition 4.3. Let $M \subset \mathbb{C}^{n+1}$ be a connected real-analytic hypersurface whose Levi form is nondegenerate at some point. Then, for every $p \in M$, there exist normal coordinates $(z, w) \in \mathbb{C}^{n} \times \mathbb{C}$, vanishing at $p$, such that $M$ near $p$ is given in these coordinates by an equation of the following form

$$
\operatorname{Im} w=\sum_{j=1}^{n} \epsilon_{j}\left|z_{j}\right|^{2}(\operatorname{Re} w)^{b_{j}} \theta_{j}(\operatorname{Re} w)+R(z, \bar{z}, \operatorname{Re} w),
$$

where each $\epsilon_{j} \in\{-1,1\}, b_{j} \in \mathbb{N}, b_{1} \geq b_{2} \geq \ldots \geq b_{n}, \theta_{j}$ is a real-analytic function near $0 \in \mathbb{R}$ satisfying $\theta_{j}(0)=1$, and $R$ is a real-analytic function near $0 \in \mathbb{R}^{2 n+1}$ satisfying

$$
R(z, 0, \operatorname{Re} w)=R(0, \bar{z}, \operatorname{Re} w) \equiv 0, \quad \text { and } \quad R(z, \bar{z}, \operatorname{Re} w)=O\left(|z|^{3}\right) .
$$

Proof. Since the set of Levi-nondegenerate points is dense in $M$, we may choose a real-analytic curve $\Gamma$ passing through $p$, transverse to $T_{p}^{c} M$, and passing through a Levi-nondegenerate point of $M$. Shrinking furthermore the curve $\Gamma$ near $p$ if necessary, we may assume that $p$ is the only point of $\Gamma$ where $M$ may be Levi-degenerate. By Lemma 4.1, we may choose normal coordinates, that we denote by $\left(z^{\prime}, w^{\prime}\right)$, vanishing at $p$, such that $M$ is given by an equation of the form

$$
\operatorname{Im} w^{\prime}=\phi\left(z^{\prime}, \bar{z}^{\prime}, \operatorname{Re} w^{\prime}\right),
$$

with $\phi$ real-analytic near the origin in $\mathbb{R}^{2 n+1}, \phi\left(z^{\prime}, 0, \operatorname{Re} w^{\prime}\right)=\phi\left(0, \bar{z}^{\prime}, \operatorname{Re} w^{\prime}\right) \equiv 0$ and such that the germ at $p$ of the curve $\Gamma$ is given by (the germ at 0 of) the real line $\{(0, s): s \in \mathbb{R}\}$. From the construction, we also know that for $s \in \mathbb{R}$ sufficiently small, the $n \times n$ matrix $A(s):=\phi_{z \bar{z}}(0, s)$ is nondegenerate for all $s \neq 0$. Since $A(s)$ is hermitian for each $s$ close to 0 , we may apply Proposition 4.2 to get the existence of a unitary matrix $U(s)$ real-analytic near the origin satisfying

$$
U(s) A(s) U^{*}(s)=\mathcal{D}(s)=\left(\begin{array}{ccc}
D_{1}(s) & 0 & 0 \\
0 & \ddots & 0 \\
0 & 0 & D_{n}(s)
\end{array}\right),
$$

with each $D_{j}, j=1, \ldots, n$, being a nonzero real-analytic function near the origin, with the origin as the only possible zero for each $D_{j}$. We now construct the desired normal 
coordinate system $(z, w)$ by defining it through the following holomorphic change of variables:

$$
\left\{\begin{array}{l}
z^{\prime}=U(w) \cdot z \\
w^{\prime}=w
\end{array}\right.
$$

Here again, we also use the notation $U$ to mean the complexification of $U$ to a sufficiently neighbourhood of 0 in $\mathbb{C}$. In the new $(z, w)$ coordinates, the germ of $M$ at 0 is given by the equation

$$
\operatorname{Im} w=\phi(U(w) \cdot z, \overline{U(w) \cdot z}, \operatorname{Re} w),
$$

or equivalently, by using the implicit function theorem by another equation of the form

$$
\operatorname{Im} w=\Phi(z, \bar{z}, \operatorname{Re} w)
$$

for some other real-analytic function $\Phi$ defined near the origin in $\mathbb{R}^{2 n+1}$. It is easy to check that $(z, w)$ are normal coordinates for $M$ i.e. that $\Phi(z, 0, \operatorname{Re} w)=\Phi(0, \bar{z}, \operatorname{Re} w) \equiv$ 0 and that the $n \times n$ matrix $\Phi_{z \bar{z}}(0, s)$ is equal to $\mathcal{D}(s)$. From this, it is not difficult to derive the final normal form given by (4.3), rescaling and interchanging the $z$ coordinates if necessary. The proof of Proposition 4.3 is therefore complete.

\section{Resolution of degeneracies via blow-ups and proof of Theorem 1.2.}

5.1. The blow-up procedure. Throughout $\S 5.1$, we fix a germ of a realanalytic hypersurface $M$ through the origin in $\mathbb{C}^{n+1}$ for which there exists a system of normal coordinates $(z, w)=\left(z_{1}, \ldots, z_{n}, w\right) \in \mathbb{C}^{n} \times \mathbb{C}$, that we also fix for the remainder of this section, such that $M$ is given by an equation of the form (4.3) with all corresponding quantities satisfying the conditions of Proposition 4.3.

Our goal here is to construct a good nonminimal real-analytic hypersurface $\widehat{M}$ (as defined in §3) contained in the pre-image of $M$ under a certain explicit blow-up. It should be mentioned that the use of blow-ups in $\mathbb{C}^{2}$ already appears for other purposes in the papers $[34,22,26]$.

The relevant statement for this paper is given by the following.

Proposition 5.1. Let $M$ be given as above and $\mathcal{B}: \mathbb{C}^{n+1} \rightarrow \mathbb{C}^{n+1}$ be the polynomial map given by

$$
\mathcal{B}(z, w):=\left(z_{1} w^{\alpha_{1}}, \ldots, z_{n} w^{\alpha_{n}}, w^{2}\right),
$$

with $\alpha_{j}:=2+3 b_{1}-b_{j}$ for $j=1, \ldots, n$. Then there exists a unique germ of a realanalytic hypersurface $\widehat{M}$ through the origin in $\mathbb{C}^{n+1}$, contained in $\mathcal{B}^{-1}(M)$, which is of the form

$$
\operatorname{Im} w=(\operatorname{Re} w)^{3+6 b_{1}} \eta(z, \bar{z}, \operatorname{Re} w),
$$

for some real-analytic function $\eta$ defined near $0 \in \mathbb{R}^{2 n+1}$. Furthermore, $\eta$ satisfies

$$
\eta(z, \bar{z}, 0)=\frac{1}{2} \sum_{j=1}^{n} \epsilon_{j}\left|z_{j}\right|^{2}, \quad \text { and } \quad \eta(z, 0, \operatorname{Re} w)=\eta(0, \bar{z}, \operatorname{Re} w)=0
$$


Proof. In what follows, we set $s:=\operatorname{Re} w$ and $t=\operatorname{Im} w$. First note that the set of points $(z, s+i t)$ close to the origin that are contained in the preimage of $(M, 0)$ under $\mathcal{B}$ is given by the equation

$$
2 s t=\sum_{j=1}^{n} \epsilon_{j}\left|z_{j}\right|^{2}\left(s^{2}-t^{2}\right)^{b_{j}}\left(s^{2}+t^{2}\right)^{\alpha_{j}} \theta_{j}\left(s^{2}-t^{2}\right)+\widetilde{R}(z, \bar{z}, s, t),
$$

with

$$
\widetilde{R}(z, \bar{z}, s, t)=R\left(z_{1} w^{\alpha_{1}}, \ldots, \bar{z}_{n} \bar{w}^{\alpha_{n}}, \operatorname{Re}\left(w^{2}\right)\right) .
$$

Setting $t=s^{3+6 b_{1}} v$, we will show that there exists a unique real-analytic function $v=v(z, \bar{z}, s)$ such that $t=s^{3+6 b_{1}} v$ satisfies (5.4). Plugging $t=s^{3+6 b_{1}} v$ in (5.4), we get the following equation

$$
\begin{aligned}
2 s^{4+6 b_{1}} v= & \sum_{j=1}^{n} \epsilon_{j}\left|z_{j}\right|^{2} s^{2 b_{j}+2 \alpha_{j}}\left(1-s^{4+12 b_{1}} v^{2}\right)^{b_{j}}\left(1+s^{4+12 b_{1}} v^{2}\right)^{\alpha_{j}} \theta_{j}\left(s^{2}-s^{6+12 b_{1}} v^{2}\right) \\
& +\widetilde{R}\left(z, \bar{z}, s, s^{3+6 b_{1}} v\right) .
\end{aligned}
$$

By our choice of $\alpha_{j}$, we have $s^{2 b_{j}+2 \alpha_{j}}=s^{4+6 b_{1}}$. To show that there exists a unique real-analytic function $v=v(z, \bar{z}, s)$ satisfying (5.6), it is enough to see that $\widetilde{R}\left(z, \bar{z}, s, s^{3+6 b_{1}} v\right)=O\left(s^{5+6 b_{1}}\right)$. We claim that one has even $\widetilde{R}\left(z, \bar{z}, s, s^{3+6 b_{1}} v\right)=O\left(s^{6+6 b_{1}}\right)$. Indeed, since $R(z, \bar{z}, \operatorname{Re} w)=O\left(|z|^{3}\right)$, we have in view of (5.5) that $\widetilde{R}(z, \bar{z}, s, t)=O\left(|w|^{3 \alpha_{1}}\right)=O\left(s^{3 \alpha_{1}}\right)=O\left(s^{6+6 b_{1}}\right)$, proving the claim. Dividing (5.6) by $s^{4+6 b_{1}}$, we get that $v$ satisfies the real-analytic equation

$$
2 v=\sum_{j=1}^{n} \epsilon_{j}\left|z_{j}\right|^{2}\left(1-s^{4+12 b_{1}} v^{2}\right)^{b_{j}}\left(1+s^{4+12 b_{1}} v^{2}\right)^{\alpha_{j}} \theta_{j}\left(s^{2}-s^{6+12 b_{1}} v^{2}\right)+S(z, \bar{z}, s, v),
$$

with $S(z, \bar{z}, s, v)=O\left(s^{2}\right)$. By the implicit function theorem, (5.7) has a unique realanalytic function solution $v=v(z, \bar{z}, s)$. This proves the first part of the proposition.

For the second part of the proposition, we first notice that $S(z, 0, s, v)=$ $\frac{\widetilde{R}\left(z, 0, s, s^{3+6 b_{1}} v\right)}{s^{4+6 b_{1}}}=0$ and similarly $S(0, \bar{z}, s, v)=0$. This proves that $\eta(z, 0, s)=$ $\eta(0, \bar{z}, s)=0$. Finally, the fact that $\eta(z, \bar{z}, 0)=\frac{1}{2} \sum_{j=1}^{n} \epsilon_{j}\left|z_{j}\right|^{2}$ clearly follows from (5.7) and the fact that $\theta_{\nu}(0)=1$ for all $\nu=1, \ldots, n$. The proof of Proposition 5.1 is complete.

We now come to the crucial property satisfied by the hypersurface $\widehat{M}$ constructed in Proposition 5.1.

Proposition 5.2. Let $\mathcal{B}, M$ and $\widehat{M}$ be as above. Then for every integer $\ell \geq$ $\max \left\{\alpha_{n}, 3+6 b_{1}\right\}$, where $\alpha_{n}$ and $b_{1}$ are given in Proposition 5.1, if $H:\left(\mathbb{C}^{n+1}, 0\right) \rightarrow$ $\left(\mathbb{C}^{n+1}, 0\right)$ is a holomorphic mapping sending $M$ into itself satisfying $j_{0}^{\ell} H=j_{0}^{\ell} \mathrm{Id}$, then there exists a unique holomorphic map $\widehat{H}:\left(\mathbb{C}^{n+1}, 0\right) \rightarrow\left(\mathbb{C}^{n+1}, 0\right)$ sending $\widehat{M}$ into itself such that $\mathcal{B} \circ \widehat{H}=H \circ \mathcal{B}$ with $j_{0}^{\ell} \widehat{H}=j_{0}^{\ell} \mathrm{Id}$.

Proof. Fix any integer $\ell \geq \max \left\{\alpha_{n}, 3+6 b_{1}\right\}$ and $H:\left(\mathbb{C}^{n+1}, 0\right) \rightarrow\left(\mathbb{C}^{n+1}, 0\right)$ a holomorphic map sending $M$ into itself satisfying $j_{0}^{\ell} H=j_{0}^{\ell} \mathrm{Id}$. We first show that 
there exists a holomorphic map $\widehat{H}:\left(\mathbb{C}^{n+1}, 0\right) \rightarrow\left(\mathbb{C}^{n+1}, 0\right)$ such that $\mathcal{B} \circ \widehat{H}=H \circ \mathcal{B}$ with $j_{0}^{\ell} \widehat{H}=j_{0}^{\ell} \mathrm{Id}$. Then we will show that the map $\widehat{H}$ is unique and sends $\widehat{M}$ into itself.

We write the map $H=(F, G) \in \mathbb{C}^{n} \times \mathbb{C}$ and similarly the desired map $\widehat{H}$ will be split as follows $(\widehat{F}, \widehat{G})$. We first note that since $(z, w)$ are normal coordinates for $M$ and since $j_{0}^{\ell} H=j_{0}^{\ell} \operatorname{Id}$, we have $G(z, w)=w(1+\Psi(z, w))$, where $\Psi$ is a convergent power series vanishing at least to order $\ell-1$. Therefore setting

$$
\widehat{G}(z, w)=w \sqrt{1+(\Psi \circ \mathcal{B})(z, w)}
$$

we have that $\widehat{G}$ satisfies

$$
\widehat{G}^{2}=G \circ \mathcal{B}, \quad \text { and } \quad \widehat{G}(z, w)=w \text {, up to order } \ell .
$$

Next, we claim that if we write $F=\left(F_{1}, \ldots, F_{n}\right)$, each power series $F_{j} \circ \mathcal{B}$ is divisible by $w^{\alpha_{j}}$ for every $j=1, \ldots, n$. Indeed, by assumption we may write $F_{j}(z, w)=z_{j}+$ $\Phi_{j}(z, w), \Phi_{j}$ being a power series vanishing at least up to order $\ell$. Hence, since $\left(\Phi_{j} \circ\right.$ $\mathcal{B})(z, w)=\Phi_{j}\left(z_{1} w^{\alpha_{1}}, \ldots, z_{n} w^{\alpha_{n}}, w^{2}\right)$ and since each $\alpha_{j} \geq 2$, we get that necessarily $w^{2(\ell+1)}$ divides $\left(\Phi_{j} \circ \mathcal{B}\right)(z, w)$ which proves the claim since $2(\ell+1)>\alpha_{n} \geq \alpha_{j}$ for each $j$. We may now set

$$
\widehat{F}_{j}(z, w):=\frac{\left(F_{j} \circ \mathcal{B}\right)(z, w)}{(\widehat{G}(z, w))^{\alpha_{j}}}
$$

which defines a convergent power series in view of (5.8) and the above claim. From (5.9) and (5.10) we automatically have $H \circ \mathcal{B}=\mathcal{B} \circ \widehat{H}$. To prove that for each $j=1, \ldots, n, F_{j}(z, w)=z_{j}$ up to order $\ell$, we write

$$
\widehat{F}_{j}(z, w)=\frac{\left(F_{j} \circ \mathcal{B}\right)(z, w)}{w^{\alpha_{j}}}(1+(\Psi \circ \mathcal{B})(z, w))^{-\alpha_{j} / 2},
$$

and notice that $\left(F_{j} \circ \mathcal{B}\right)(z, w) / w^{\alpha_{j}}$ agrees with $z_{j}$ up to order (at least) $2(\ell+1)-1-\alpha_{j} \geq$ $2 \ell+1-\alpha_{n}$ and that $(1+(\Psi \circ \mathcal{B})(z, w))^{-\alpha_{j} / 2}$ agrees with 1 up to order (at least) $2 \ell-1$. From this we conclude that $\widehat{F}_{j}(z, w)$ agrees with $z_{j}$ up to order at least $2 \ell+1-\alpha_{n} \geq \ell$, which proves that $j_{0}^{\ell} \widehat{H}=j_{0}^{\ell} \mathrm{Id}$.

The uniqueness of $\widehat{H}$ is clear since there are only two possibles choices for constructing a power series $T(z, w)$ such that $T^{2}=G \circ \mathcal{B}$ i.e. $T= \pm \widehat{G}$ with $\widehat{G}$ as constructed above. Since we require $\widehat{H}$ to have the same $\ell$-jet as that of the identity mapping with $\ell \geq \alpha_{n} \geq 1, T=\widehat{G}$ is the only possible choice for the normal component of $\widehat{H}$. This finally also implies the uniqueness of the other components of $\widehat{H}$ since they must satisfy (5.10).

It remains to check that the constructed holomorphic map $\widehat{H}:\left(\mathbb{C}^{n+1}, 0\right) \rightarrow$ $\left(\mathbb{C}^{n+1}, 0\right)$ indeed sends $\widehat{M}$ into itself. For this we first note that $M^{\prime}:=\widehat{H}(\widehat{M})$ defines a real-analytic hypersurface that is of infinite type since $\widehat{M}$ is. Secondly, since $H$ sends $M$ into itself and $\mathcal{B} \circ \widehat{H}=H \circ \mathcal{B}$, we have that $\widehat{H}(\widehat{M}) \subset \mathcal{B}^{-1}(M)$. We now claim that $M^{\prime}$ is a real-analytic hypersurface of the form $\operatorname{Im} w=(\operatorname{Re} w)^{3+6 b_{1}} \rho(z, \bar{z}, \operatorname{Re} w)$ for some convergent power series $\rho$. From the claim, one gets that $M^{\prime}=\widehat{M}$ since $\widehat{M}$ is the unique real-analytic hypersurface of the above form contained in $\mathcal{B}^{-1}(M)$ by Proposition 5.1. Now, the proof of the claim is easily obtained by using the following fact that follows from our construction of the map $\widehat{H}$ : one has $\widehat{G}(z, w)=w+w^{2(\ell+1)} \delta(z, w)$ 
for some power series $\delta$. Since $2(\ell+1)>3+6 b_{1}$, a direct computation of $\widehat{H}(\widehat{M})$, left to the reader, shows that it has the form claimed above. The proof of Proposition 5.2 is complete.

Recall that $M$ (resp. $\widehat{M}$ ) has the finite jet determination property at 0 if there exists a positive integer $r$ such that if $H \in \operatorname{Aut}(M, 0)$ (resp. $H \in \operatorname{Aut}(\widehat{M}, 0))$ agrees with the identity mapping at the origin up to order $r$, then $H$ is the identity. As an immediate consequence of Proposition 5.2, we obtain the following crucial result.

Corollary 5.3. If $\widehat{M}$ has the finite jet determination property at 0 , so does $M$.

REMARK 5.4. If, throughout this section, $M$ is a formal real hypersurface (instead of a real-analytic one), still of the form (4.3) with $R$ and each $\theta_{j}$ being formal power series, then Propositions 5.1 and 5.2 have a formal counterpart. The formal version of Proposition 5.1 will provide a unique formal real hypersurface $\vec{M}$ of the form (5.2) with $\eta$ being a formal power series satisfying also (5.3). In addition, the lifting procedure given by Proposition 5.2 also holds in that setting for formal holomorphic maps $H$. The proofs of these statements are obtained by mimicing the proofs given here in a formal setting. The details are left to the reader.

5.2. Proof of Theorem 1.2. The proof of the theorem is achieved by combining Proposition 4.3, Proposition 5.1, Corollary 5.3 and Proposition 3.2.

\section{REFERENCES}

[1] M. S. Baouendi, P. Ebenfelt, And L. P. Rothschild, CR automorphisms of real analytic manifolds in complex space, Comm. Anal. Geom., 6:2 (1998), pp. 291-315.

[2] M. S. Baouendi, P. Ebenfelt, And L. P. Rothschild, Real submanifolds in complex space and their mappings, Princeton University Press, Princeton, NJ, 1999.

[3] M. S. Baouendi, P. Ebenfelt, And L. P. Rothschild, Convergence and finite determination of formal CR mappings, J. Amer. Math. Soc., 13:4 (2000), pp. 697-723 (electronic).

[4] M. S. Baouendi, P. Ebenfelt, and L. P. Rothischild, Local geometric properties of real submanifolds in complex space, Bull. Amer. Math. Soc. (N.S.), 37:3 (2000), pp. 309-336 (electronic).

[5] M. S. Baouendi, X. Huang, And L. P. Rothschild, Regularity of CR mappings between algebraic hypersurfaces, Invent. Math., 125:1 (1996), pp. 13-36.

[6] M. S. Baouendi, N. Mir, And L. P. Rothschild, Reflection ideals and mappings between generic submanifolds in complex space, J. Geom. Anal., 12:4 (2002), pp. 543-580.

[7] V.K. Beloshapka, On the dimension of the group of automorphisms of an analytic hypersurface, Math. Notes, 14 (1980), pp. 223-245.

[8] V.K. BeloshapkA, A uniqueness theorem for automorphisms of a nondegenerate surface in a complex space, Math. Notes, 47:3 (1990), pp. 239-242.

[9] E. CARTAN, Sur la géométrie pseudo-conforme des hypersurfaces de deux variables complexes I, Ann. Math. Pura Appl., 11:4 (1932), pp. 17-90.

[10] E. CARTAn, Sur la géométrie pseudo-conforme des hypersurfaces de deux variables complexes II, Ann. Scuola Norm. Sup. Pisa, 1:2 (1932), pp. 333-354.

[11] H. Cartan, Sur les groupes de transformations analytiques, Act. Sc. et Int. Hermann, Paris, 1935.

[12] S. S. Chern And J. K. Moser, Real hypersurfaces in complex manifolds, Acta Math., 133 (1974), pp. 219-271.

[13] P. EBenfelt, Finite jet determination of holomorphic mappings at the boundary, Asian J. Math., 5:4 (2001), pp. 637-662.

[14] P. EBenfelt, On the analyticity of CR mappings between nonminimal hypersurfaces, Math. Ann., 322:3 (2002), pp. 583-602.

[15] P. Ebenfelt and B. Lamel, Finite jet determination of CR embeddings, J. Geom. Anal., 14:2 (2004), pp. 241-265. 
[16] P. Ebenfelt, B. LAmel, And D. Zaitsev, Finite jet determination of local analytic CR automorphisms and their parametrization by 2-jets in the finite type case, Geom. Funct. Anal., 13:3 (2003), pp. 546-573.

[17] H. Gingold And P. F. Hsien, Globally analytic triangularization of a matrix function, Linear Algebra Appl., 169 (1992), pp. 75-101.

[18] C.-K. HAN, Complete differential system for the mappings of CR manifolds of nondegenerate Levi forms, Math. Ann., 309:3 (1997), pp. 401-409.

[19] S.-Y. KIM, Complete system of finite order for CR mappings between real analytic hypersurfaces of degenerate Levi form, J. Korean Math. Soc., 38:1 (2001), pp. 87-99.

[20] S. Y. Kim AND D. ZAITSEV, Equivalence and embedding problems for CR-structures of any codimension, Topology, 44:3 (2005), pp. 557-584.

[21] R. T. KowALSkI, A hypersurface in $\mathbb{C}^{2}$ whose stability group is not determined by 2-jets, Proc. Amer. Math. Soc., 130:12 (2002), pp. 3679-3686 (electronic).

[22] R.T. KowALSKI, Formal equivalences between real-analytic hypersurfaces, PhD thesis, University of California, San Diego, 2002.

[23] B. LAMEL, Holomorphic maps of real submanifolds in complex spaces of different dimensions, Pacific J. Math., 201:2 (2001), pp. 357-387.

[24] B. Lamel, Explicit bounds for the finite jet determination problem, Trans. Amer. Math. Soc., 358 (2006), pp. 3447-3457.

[25] B. Lamel AND N. Mir, Finite jet determination of CR mappings, Adv. Math. (2007), doi:10.1016/j.aim.2007.05.007.

[26] B. Lamel And N. Mir, Remarks on the rank properties of formal CR maps, Sci. China, Ser. A, 49:11 (2006), pp. 1477-1490.

[27] B. Lamel AND N. MiR, Parametrization of local CR automorphims by finite jets and applications, J. Amer. Math. Soc, 20:2 (2007), pp. 519-572.

[28] A. V. Loboda, On local automorphisms of real-analytic hypersurfaces, Math. USSR, Izv., 18 (1982), pp. 537-559.

[29] F. Meylan, A reflection principle in complex space for a class of hypersurfaces and mappings, Pacific J. Math., 169:1 (1995), pp. 135-160.

[30] F. Rellich, Perturbation Theory of Eigenvalue Problems, Gordon and Breach, New York, 1969.

[31] L.P. RothSChILD, Iterated Segre mappings of real submanifolds in complex space and applications, International Congress of Mathematicians. Vol. II, pp. 1405-1419, Eur. Math. Soc., Zürich, 2006.

[32] N. K. Stanton, Infinitesimal CR automorphisms of real hypersurfaces, Amer. J. Math., 118:1 (1996), pp. 209-233.

[33] N. TANAKA, On the pseudo-conformal geometry of hypersurfaces of the space of $n$ complex variables, J. Math. Soc. Japan, 14 (1962), pp. 397-429.

[34] D. Zaitsev, Unique determination of local CR-maps by their jets: A survey, Atti Accad. Naz. Lincei Cl. Sci. Fis. Mat. Natur. Rend. Lincei (9) Mat. Appl., 13 (2002), pp. 295-305. 